\title{
Estrategias de enseñanza docente en la satisfacción académica de los estudiantes universitarios
}

\author{
Teaching strategies in university students' academic satisfaction
}

\author{
Americo Hurtado-Palomino ${ }^{1, a}$ 0000-0003-2293-9927 \\ Willam Merma-Valverde 1,b 0000-0003-0290-9560 \\ Flor de Meliza Ccorisapra-Quintana ${ }^{2, \mathrm{c}}$ 0000-0002-6225-7296 \\ Yarixa Lazo-Cerón ${ }^{1, \mathrm{~d}}$ 0000-0002-3661-2004 \\ Karol Boza-Salas ${ }^{1, e}$ 0000-0001-8763-058X \\ ${ }^{1}$ Universidad Nacional de San Agustín de Arequipa, Arequipa, Perú. \\ ${ }^{2}$ Universidad Tecnológica del Perú, Perú. \\ a ahurtado@unsa.edu.pe \\ bwmermav@unsa.edu.pe \\ cc21383@utp.edu.pe \\ dylazo@unsa.edu.pe \\ e kboza@unsa.edu.pe
}

Recibido: 24/07/2021
Aprobado: 26/08/2021
Publicado: 15/09/2021

\section{Resumen}

El actual escenario universitario exige mayor planificación y desarrollo de estrategias de enseñanza docente que permitan alcanzar un óptimo aprendizaje y satisfacción académica. El presente artículo tiene como objetivo analizar el efecto de las estrategias de enseñanza docente a través de la estrategia instrumental, afectivomotivacional y cognitiva-metacognitiva en la satisfacción del estudiante universitario en un entorno de aprendizaje virtual sincrónico. La metodología del estudio, sigue la ruta del enfoque cuantitativo, de alcance explicativo, para el cual se realiza 469 encuestas a estudiantes de pregrado. Además, para examinar los datos se utiliza la regresión lineal múltiple. Los resultados muestran que todas las dimensiones de las estrategias de enseñanza tienen una relación directa positiva en la satisfacción académica. Sin embargo, la dimensión afectivo-motivacional tiene mayor incidencia que las demás estrategias. Finalmente, se concluye que los docentes aplican estrategias de enseñanza en un entorno de aprendizaje sincrónico de manera satisfactoria.

Palabras clave: aprendizaje sincrónico; estrategias de enseñanza; estudiantes universitarios; satisfacción académica.

\begin{abstract}
The current university scenario requires more planning and development of teaching strategies to achieve optimal learning and academic satisfaction. The aim of this article is to analyse the effect of teaching strategies through instrumental, affectivemotivational and cognitive-metacognitive strategies on university student satisfaction in a synchronous virtual learning environment. The methodology of the study, follows the route of the quantitative approach, of explanatory scope, for which 469 surveys are conducted to undergraduate students. In addition, multiple linear regression is used to examine the data. The results show that all dimensions of teaching strategies have a direct positive relationship with academic satisfaction. However, the affective-motivational dimension has a higher incidence than the other strategies. Finally, it is concluded that teachers apply teaching strategies in a synchronous learning environment in a satisfactory way.
\end{abstract}

Keywords: synchronous learning; teaching strategies; university students; academic satisfaction. 


\section{Introducción}

Las consecuencias de la COVID-19 han puesto a prueba el proceso de enseñanza-aprendizaje en las instituciones universitarias, adaptándose casi inmediatamente a las tecnologías digitales para continuar con las actividades académicas y ofrecer una formación universitaria sin mermar los estándares de calidad (Candrlic et al., 2020). En ese sentido, el cambio a la modalidad de enseñanza virtual sincrónica, exige un conjunto de competencias y habilidades docentes, como el uso de redes y plataformas digitales, que sirven de apoyo en la labor docente, además, permite una mayor interacción entre los docentes y estudiantes (Cabanillas et al., 2020).

En este contexto, se ha valorado el rol del docente universitario (Beardsley et al., 2021) a través del empleo de estrategias de enseñanza, considerando un conjunto de métodos aplicados de manera intencional, reflexiva y flexible para el aprendizaje significativo de los estudiantes (Henríquez y Arámburo, 2019). Además, en el proceso de enseñanza se desarrolla las competencias cognitivas y habilidades profesionales, orientando cada actividad al logro de mayor satisfacción académica del estudiante (Betancourt et al., 2020). En este sentido, se requiere el compromiso del docente para alcanzar la sostenibilidad de la calidad educativa (Hurtado et al., 2021).

Al revisar la literatura previa sobre las estrategias de enseñanza docente y la satisfacción académica, los resultados muestran escasos estudios empíricos. Sin embargo, existen estudios que relacionan la motivación, inteligencia emocional del docente, éxito académico, calidad de los servicios ofrecidos por la universidad, prácticas docentes y las características de cada asignatura con la satisfacción académica (Gonzales et al., 2019; Candrlic et al., 2020; Tacca et al., 2020; Zambrano, 2020; Llanes et al., 2021). Al respecto, los estudios desarrollados por Zambrano (2020) y Tacca et al. (2020) sostienen que los factores de inteligencia emocional del docente muestran una correlación positiva con la satisfacción académica del estudiante. Por otro lado, Martínez et al. (2018) concluyen que las estrategias de enseñanza superior online desarrollan conocimiento y habilidades docentes con dominio conceptual, social, cultural y tecnológico, claves en el proceso de enseñanza virtual. En esta línea, Gonzales et al. (2019) sostienen que la satisfacción académica varía según la especialidad y los niveles académicos. Posteriormente, Llanes et al. (2021) sostienen profundizar sobre los antecedentes de la satisfacción académica en diferentes contextos geográficos. Además, Candrlic et al. (2020) sugieren la necesidad de conocer y entender las expectativas de los estudiantes para el adecuado desarrollo del plan de estudios con el fin de incrementar su satisfacción.

No obstante, ante esta revisión del estado del arte, se evidencia que existe un vacío de literatura en relación a la vinculación directa de las estrategias de enseñanza docente con la satisfacción académica, asimismo, se evidencia la ausencia de investigaciones que desarrollen a profundidad la categorización de las dimensiones presentadas por Zambrano et al. (2015). En tal sentido, se rescata la importancia de la presente investigación, pues forma parte de los primeros trabajos en presentar una vinculación entre estas variables, así como su relación en el proceso de enseñanza-aprendizaje. Frente a esta demanda, se requiere explorar en mayor detalle sobre las causas de la satisfacción académica de los estudiantes que permita tener una mejor experiencia universitaria.

La presente investigación tiene por objetivo analizar el efecto de las estrategias de enseñanza docente a través de sus dimensiones: instrumental, afectivo-motivacional y cognitiva-metacognitiva en la satisfacción académica del estudiante universitario. Esta investigación contribuye de manera teórica y empírica, los efectos directos de las dimensiones de las estrategias de enseñanza en la satisfacción académica. Además, se convierte en un precedente en el ámbito pedagógico y futuras líneas de investigación. En cuanto a la estructura, es desarrollada de la siguiente manera, se inicia con la introducción; luego, el marco teórico realizándose una revisión bibliográfica que explican los fundamentos teóricos acerca de la satisfacción académica, estrategias de enseñanza docente y las dimensiones educativas con sus respectivas relaciones propuestas. Seguidamente, se presenta la metodología, resultados y discusión. Finalmente, se aborda las conclusiones, limitaciones y futuras líneas de investigación. 


\section{Marco teórico}

\section{Estrategias de enseñanza docente}

La actividad realizada por el docente en el aula ha ganado relevancia en los últimos años por su contribución en el proceso de aprendizaje. Asimismo, Zambrano et al., (2015) consideran que las estrategias docentes, también fueron estudiadas como "estrategias didácticas, modalidades de enseñanza, estilos de enseñanza (...)" (p.27). En este estudio, se ha optado por analizar como estrategia de enseñanza docente, entendida como el uso de diversos recursos de ayuda pedagógica en el desarrollo de la actividad académica. Sin embargo, Henríquez y Arámburo (2019) entienden como un conjunto de métodos aplicados por el docente de manera intencional, reflexiva y flexible, para el aprendizaje significativo de los estudiantes. Además, aportan una infinidad de opciones para obtener óptimas condiciones de enseñanza, desarrollar el potencial de cada estudiante y fortalecer el proceso de enseñanza (Betancourt et al., 2020). En general, las estrategias de enseñanza orientan la actividad del docente universitario para alcanzar mayor satisfacción del estudiante.

Para Zambrano et al. (2015) las estrategias de enseñanza docente se deben evaluar a través de las siguientes dimensiones: instrumental, afectivomotivacional y cognitiva-metacognitiva, dado que, comprender la importancia de estas dimensiones, permite al docente universitario obtener una mejor perspectiva al momento de planear y ejecutar el proceso de enseñanza-aprendizaje, asimismo, en la literatura más reciente se observa un mayor interés por estudiar la efectividad de las estrategias docentes, lo que supone ofrecer información sobre lo que sucede dentro del salón de clase y ampliar el campo de investigación en las estrategias de enseñanza docente universitarias (Gil et al., 2017).

\section{Satisfacción académica}

En esta investigación la satisfacción académica es estudiada desde la perspectiva del bienestar psicológico, la cual se conceptualiza como el placer, gusto o deleite que siente el estudiante por sus quehaceres académicos dentro de una carrera, con la que se identifica y busca desarrollar sus habilidades, además, mantiene una relación directa con la metodología de enseñanza en la integración social, persistencia académica y explicación de experiencias educativas como la adaptación, bienestar, desempeño y éxito académico (Vergara et al., 2018).

Se debe tener en consideración que la satisfacción académica depende en gran medida del clima motivacional, calidad docente, estructura curricular, infraestructura y servicios ofrecidos por la universidad; sin embargo, la mayoría de tales elementos logran evidenciar la relevancia del papel docente como uno de los agentes más importantes en el proceso de enseñanza-aprendizaje, dado que con sus acciones y actitudes influyen en la satisfacción del estudiante. En ese sentido, se destaca la idea de vincular las estrategias de enseñanza docente, con tal variable, pues captar la atención del estudiante, evitar el abandono y, principalmente garantizar la calidad académica, forman parte de los objetivos de la educación universitaria (Ortega et al., 2019).

\section{Estrategia instrumental y satisfacción académica}

Esta estrategia es estudiada a través de dos indicadores: planificación didáctica y ambiente en el salón de clase; en el primer caso, evalúa el modelo pedagógico desarrollado por el docente a través de diferentes conceptos y materiales de estudios; en la segunda, hace referencia al clima generado por los vínculos interpersonales, aceptación de las diferencias entre personas, ideas y otros, establecidos por normas claras implementadas por el docente para el correcto desarrollo de la sesión sincrónica, además, influye en la disposición de los estudiantes en participar activamente, cuanto más óptimo sea el ambiente del aula, hay más disposición de aprender y estudiar, al mismo tiempo que los docentes estén dispuestos a enseñar (Zambrano et al., 2015). Además, vincular la estrategia instrumental con la satisfacción académica se debe por su rol en el proceso de enseñanza-aprendizaje, pues involucra el dominio de conocimientos que permitan la participación de los estudiantes, por ello, esta estrategia es definida como el conjunto de conocimientos y herramientas fundamentales que permiten adquirir el resto de aprendizajes, he ahí la relevancia del desempeño del docente pues, independientemente del ámbito donde se desarrolle, es necesario que reflexione el que va hacer, como lo hará, que herramientas utilizará y en dónde lo va a desarrollar (Abreu et al., 2018).

Tales argumentos sugieren inferir que el clima o ambiente en el salón de clases afecta directamente 
en la satisfacción y resultado académico del estudiante.

H1: La estrategia instrumental tiene un efecto directo positivo en la satisfacción académica de los estudiantes universitarios.

\section{Estrategia afectivo-motivacional y satisfacción académica}

La estrategia afectivo-motivacional permite lograr una eficiente labor docente, dado que fortalece las capacidades del estudiante en beneficio del rendimiento académico (Iglesias, 2016). En esta línea, Zambrano et al. (2015) destacan dos indicadores; la estrategia evaluativa, la cual se relaciona con la valoración de aportes, versatilidad y criterios de calificación; y la estrategia de mediación emocional, que enfatiza el clima de expresión, intercambio de ideas y una retroalimentación positiva en la sesión de clases sincrónica.

Dentro de este marco, Hortigüela et al. (2017) sostienen que la satisfacción académica está relacionada a los procedimientos de evaluación y calificación estructurados, a partir de criterios y metodologías abiertas que favorezcan la implicación del estudiante en su proceso de aprendizaje y de retroalimentación inmediata, así como su valoración relacionada al docente como persona más que en lo pedagógico y cognitivo. Asimismo, Iglesias (2016) identificó en su investigación características vinculadas a la satisfacción académica en base a la estrategia afectivo-motivacional, donde las variables más valoradas fueron: “(...) que el docente sea amigable, cercano y accesible (...)", con la mayor cantidad de estudiantes que reconocen a esta variable como indicador de satisfacción, y, “(...) que sean conscientes de su labor como educador (...)" fue la variable con menor reconocimiento (p. 80). A partir de estos argumentos se ha formulado la siguiente hipótesis de investigación.

H2: La estrategia afectivo-motivacional tiene un efecto directo positivo en la satisfacción académica de los estudiantes universitarios.

\section{Estrategia cognitiva-metacognitiva satisfacción académica}

En referencia a la estrategia cognitivametacognitiva, Zambrano et al. (2015) consideran dos estrategias para su estudio; la mediación cognitiva, la cual permite desarrollar la capacidad cognitiva-metacognitiva; y los recursos didácticos, dónde destaca el uso y diversidad de las tecnologías de apoyo, con el objetivo de alcanzar las competencias del curso. En este sentido, se reconoce la importancia de la capacitación e instrucción a los estudiantes en el manejo de plataformas virtuales para fortalecer su aprendizaje.

Esta estrategia permite integrar la información que se adquiere a través de los sentidos para crear estructuras del conocimiento, donde los estudiantes desarrollan habilidades para la adquisición, organización, retención y uso del conocimiento (Orozco et al., 2019). Asimismo, Klimenko et al. (2014) evidencian la relación entre la estrategia cognitiva-metacognitiva y la satisfacción académica, cuando las dudas del estudiante son atendidas, dado que, lo motivan a acercarse más en su proceso de aprendizaje y reflexión de lo aprendido.

H3: La estrategia cognitivo-metacognitivo tiene un efecto directo positivo en la satisfacción académica de los estudiantes universitarios.

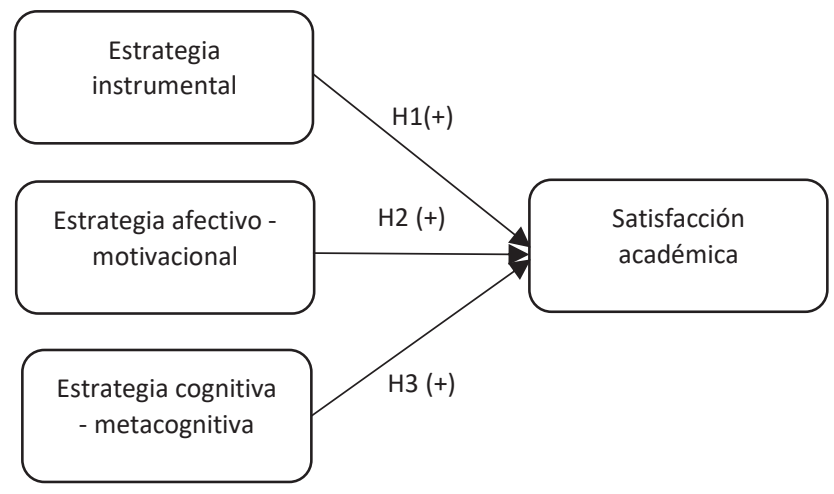

Figura 1. Modelo teórico

La figura 1, presenta el modelo teórico del estudio, donde $\mathrm{H} 1 \quad(+)$ representa la influencia directa de la estrategia instrumental en la satisfacción académica. La hipótesis $\mathrm{H} 2(+)$ representa la influencia directa de la estrategia afectivomotivacional en la satisfacción. Finalmente, la hipótesis $\mathrm{H} 3(+)$ representa la influencia directa de la estrategia cognitiva-metacognitiva en la satisfacción académica. 


\section{Materiales y métodos}

\section{Diseño}

La investigación es de enfoque cuantitativo, la ruta cuantitativa es considerada la más adecuada para contrastar las hipótesis de estudios a través de datos estadísticos (Hernández y Mendoza, 2018). Asimismo, el estudio es no experimental por su naturalezay propósitodeinvestigación. Finalmente, la investigación tiene un alcance descriptivo - correlacional - explicativa, característica de los estudios de perspectiva cuantitativa, con el propósito de recolectar información de manera independiente y en conjunto sobre las variables planteadas, así como, medir el grado de relación y sustentación de hipótesis.

\section{Población y muestra}

La población de estudio está conformada por 3298 estudiantes de pregrado matriculados en setiembre del 2020 de las facultades de administración y contabilidad - finanzas de una universidad pública, donde el principal criterio de inclusión se sostiene en la poca especialización en la metodología de enseñanza - aprendizaje de los docentes de esta área del conocimiento. La recogida de la información se realizó a través de un cuestionario estructurado para medir las variables a través de escalas validadas, de esta manera, se obtuvo un total de 469 cuestionarios válidos, que representa una tasa de respuesta del $15.38 \%$, considerando un nivel de confianza de $95 \%$, la situación favorable y desfavorable es de $\mathrm{p}=\mathrm{q}=0.5$, y el error muestral de del $4.19 \%$. Cabe recalcar que, en todo momento se ha garantizado la confidencialidad de las respuestas y la participación de los estudiantes fue de manera voluntaria.

\section{Medición: instrumentos de recolección de datos}

Para determinar las estrategias de enseñanza docente, utilizadas en el proceso de aprendizaje del estudiante se han tomado las escalas validadas por Zambrano et al. (2015), quienes miden la variable mediante tres dimensiones: Instrumental, afectivomotivacional, cognitiva-metacognitiva. En primer lugar, la estrategia instrumental, evaluadas por el ambiente en el salón de clase y planificación, fueron medidas con 9 ítems (alfa de Cronbach $=0.935)$; seguido por la estrategia afectivomotivacional, caracterizada por los indicadores evaluativas y mediación emocional, medidas con
16 ítems (alfa de Cronbach $=0.913$ ) y finalmente, la estrategia cognitiva-metacognitiva, evaluadas con los indicadores de mediación cognitiva y aspectos metacognitivos, medidas con 10 ítems (alfa de Cronbach $=0.954$ ). Todas las dimensiones superan el nivel mínimo requerido de 0.70 (George y Mallery, 2019).

Mientras que, la variable satisfacción académica, se ha medido de manera unidimensional con 7 ítems adaptados de Vergara et al. (2018). El alfa de Cronbach para esta variable es 0.938 , superando el mínimo requerido de 0.70 (George y Mallery, 2019). Ambos cuestionarios fueron preparados bajo una escala de calificación (intervalo) de siete puntos, que va de 1 (totalmente en desacuerdo) a 7 (totalmente de acuerdo), considerado adecuado por la alta sensibilidad (Hernández y Mendoza, 2018).

La tabla 1, muestra la consistencia interna de los instrumentos. Asimismo, las variables se encuentran por encima de 0.70 , por tanto, el valor es aceptable (George y Mallery, 2019), asimismo, en la tabla 2, se evidencia los valores para determinar el coeficiente alfa de Cronbach.

Tabla 1. Resumen del alfa de Cronbach.

\begin{tabular}{|c|c|c|}
\hline Variables & Dimensiones & $\begin{array}{l}\text { Alfa de } \\
\text { Cronbach }\end{array}$ \\
\hline \multirow{3}{*}{$\begin{array}{l}\text { Estrategias de } \\
\text { enseñanza } \\
\text { docente }\end{array}$} & Estrategia instrumental & 0.94 \\
\hline & $\begin{array}{l}\text { Estrategia afectivo- } \\
\text { Motivacional }\end{array}$ & 0.95 \\
\hline & $\begin{array}{l}\text { Estrategia cognitiva } \\
\text { metacognitiva }\end{array}$ & 0.97 \\
\hline $\begin{array}{l}\text { Satisfacción } \\
\text { académica }\end{array}$ & & 0.94 \\
\hline
\end{tabular}

Tabla 2. Resumen del alfa de Cronbach.

\begin{tabular}{ll}
\hline Criterio & Calificación \\
\hline$\alpha>0.9$ & Excelente \\
$\alpha>0.8$ & Bueno \\
$\alpha>0.7$ & Aceptable \\
$\alpha>0.6$ & Cuestionable \\
$\alpha>0.5$ & Pobre \\
$\alpha<0.5$ & Inaceptable \\
\hline
\end{tabular}

Nota. El baremo se ha tomado de George y Mallery (2019).

\section{Procedimiento de análisis de datos}

Los datos fueron recogidos de manera online a través del Google forms y procesados en el SPSS versión 24. Asimismo, en el análisis descriptivo se determinaron las características de la unidad de 
estudio y el nivel de percepción de las dimensiones de las estrategias de enseñanza docente y satisfacción académica. En cuanto al análisis de la estadística inferencial, en un primer momento se ha normalizado los datos (George y Mallery, 2019), luego se ha determinado las correlaciones entre las variables de estudio y finalmente, se procedió a calcular la influencia de las dimensiones de las estrategias de enseñanza docente en la satisfacción académica a través de la regresión lineal jerárquica.

\section{Resultados}

\section{Resultados descriptivos}

En este primer apartado se presenta el análisis descriptivo de los resultados de la investigación, donde se muestra el nivel de percepción de las estrategias instrumentales, afectivo-motivacional, cognitivo-metacognitivo y de la variable, satisfacción académica, donde la tabla 3 , muestra que el $78.5 \%$ de los estudiantes universitarios de negocios consideran que la estrategia instrumental percibida es alta; asimismo, el $78.9 \%$ de los encuestados consideran que el nivel de la estrategia afectivo - motivacional percibida es alto; en cuanto a la estrategia cognitiva - metacognitiva, el $75.9 \%$ de los estudiantes consideran tener una percepción de nivel alto. Finalmente, el $80 \%$ de los encuestados califican tener alta la satisfacción académica.
Tabla 3. Resultados descriptivos.

\begin{tabular}{llll}
\hline Dimensión / variable & Nivel & Frecuencia & Porcentaje \\
\hline \multirow{3}{*}{ Estrategia Instrumental } & Baja & 4 & $1 \%$ \\
& Media & 97 & $21 \%$ \\
& Alta & 368 & $79 \%$ \\
\hline \multirow{3}{*}{ Afectivo - motivacional } & Bajo & 7 & $2 \%$ \\
& Media & 92 & $20 \%$ \\
& Alta & 370 & $79 \%$ \\
\hline \multirow{3}{*}{ Cognitiva - metacognitiva } & Baja & 6 & $1 \%$ \\
& Media & 107 & $23 \%$ \\
& Alta & 356 & $76 \%$ \\
\hline \multirow{3}{*}{ Satisfacción académica } & Baja & 5 & $1 \%$ \\
& Media & 89 & $19 \%$ \\
& Alta & 375 & $80 \%$ \\
\hline
\end{tabular}

\section{Resultados inferenciales}

La tabla 4, presenta la media, desviación estándar y la correlación bivariado. Los resultados muestran que el promedio de tiempo estudiado es de 5 semestres (mínimo 1 y máximo 11). Asimismo, la edad promedio es 21 años aproximadamente (mínimo 17 y máximo 52). Además, en el análisis de correlación se encontró la correlación positiva considerable y significativa entre la estrategia instrumental y la satisfacción académica $\left(\mathrm{r}=0.713^{* *} ; \mathrm{p}<0.05\right)$. También, la estrategia afectivo-motivacional tiene una relación positiva considerable y significativa con la satisfacción académica $\left(\mathrm{r}=0.740^{* *} ; \mathrm{p}<0.05\right)$. Finalmente, la estrategia cognitiva - metacognitiva tiene relación positiva considerable y significativa con la satisfacción académica $\left(\mathrm{r}=0.740^{* *} ; \mathrm{p}<0.05\right)$. En general, se observa que las variables de estudio tienen una correlación positiva en todos los casos (Hernández y Mendoza, 2018).

Tabla 4. Resultados descriptivos y correlacionales.

\begin{tabular}{lllllllll}
\hline & Media & S.D. & $\mathbf{1}$ & $\mathbf{2}$ & $\mathbf{3}$ & $\mathbf{4}$ & $\mathbf{5}$ & $\mathbf{6}$ \\
\hline 1. Semestres estudiados & 5.03 & 2.81 & 1 & & & & & \\
2. Edad & 20.9 & 3.25 & $.410^{* *}$ & 1 & & & & \\
3. Estrategia instrumental & 5.79 & 0.93 & -0.04 & -0.03 & 1 & & & \\
4. Afectivo - motivacional & 5.66 & 0.97 & 0.002 & 0.002 & $.890^{* *}$ & 1 & & \\
5. Cognitiva - metacognitiva & 5.92 & 0.95 & -0.07 & -0.05 & $.883^{* *}$ & $.914^{* *}$ & 1 & \\
6. Satisfacción académica & 5.80 & 0.95 & -0.01 & -0.003 & $.713^{* *}$ & $.740^{* *}$ & $.732^{* *}$ & 1 \\
\hline Nota. $* \mathrm{p}<0.1 ; * * \mathrm{p}<0.05 ; * * * \mathrm{p}<0.01 ; * * * * \mathrm{p}<0.001$ & & & & &
\end{tabular}

Los resultados de la prueba de Durbin - Watson y los valores del factor de inflación de la varianza (VIF) determinan que la investigación no presentan problemas de colinealidad. El resultado de Durbin
- Watson es 2.124, encontrándose entre 1.5 y 2.5 , por tanto, no existe el problema de autocorrelación. Asimismo, los valores del VIF son menores a 10 , aceptándose los resultados. 
Tabla 5. Análisis de regresión múltiple.

\begin{tabular}{llllll}
\hline $\begin{array}{l}\text { Modelo: R2 Ajustado }= \\
\mathbf{0 . 5 6 9}\end{array}$ & \multicolumn{2}{l}{$\begin{array}{l}\text { Coeficientes no } \\
\text { estandarizados }\end{array}$} & $\begin{array}{l}\text { Coeficientes } \\
\text { estandarizados }\end{array}$ & & Sig. \\
\cline { 2 - 5 } & $\mathbf{B}$ & $\begin{array}{l}\text { Error } \\
\text { estándar }\end{array}$ & Beta $(\boldsymbol{\beta})$ & & \\
\hline (Constante) & $5.785 \mathrm{E}-07$ & 0.03 & & 0.00 & 1.00 \\
Estrategia instrumental & 0.18 & 0.07 & 0.17 & 2.40 & 0.02 \\
Afectivo - motivacional & 0.34 & 0.08 & 0.34 & 4.13 & 0.00 \\
Cognitiva - metacognitiva & 0.27 & 0.08 & 0.27 & 3.29 & 0.001 \\
\hline
\end{tabular}

Nota. Variable dependiente: Satisfacción académica.

En la tabla 5, se muestra el modelo de regresión lineal múltiple, que explica la influencia de las dimensiones de las estrategias de enseñanza docente en la satisfacción académica. Asimismo, los resultados muestran una influencia positiva y significativa de la estrategia instrumental en la satisfacción académica $(\beta=0.178, \mathrm{p}<0.05)$, lo que nos permite aceptar la hipótesis específica 1 . La dimensión afectivo - motivacional muestran una influencia positiva y significativa en la satisfacción académica $(\beta=0.344, p<0.05)$, lo que permite aceptar la hipótesis específica 2. Finalmente, la dimensión cognitiva-metacognitiva tiene influencia positiva y significativa en la satisfacción académica $(\beta=0.266, p<0.01)$, este resultado permite aceptar la hipótesis específica 3. Por otro lado, el resultado de la prueba t de Student, explica que la dimensión afectivo motivacional tiene mayor contribución y el más importante $(\mathrm{t}=4.126 ; \mathrm{p}=0.000)$; seguido por la dimensió $\mathrm{n}$ cognitiva-metacognitiva $(\mathrm{t}=0.266 ; \mathrm{p}=0.001)$ y la dimensión instrumental $(\mathrm{t}=2.403 ; \mathrm{p}=0.017)$.

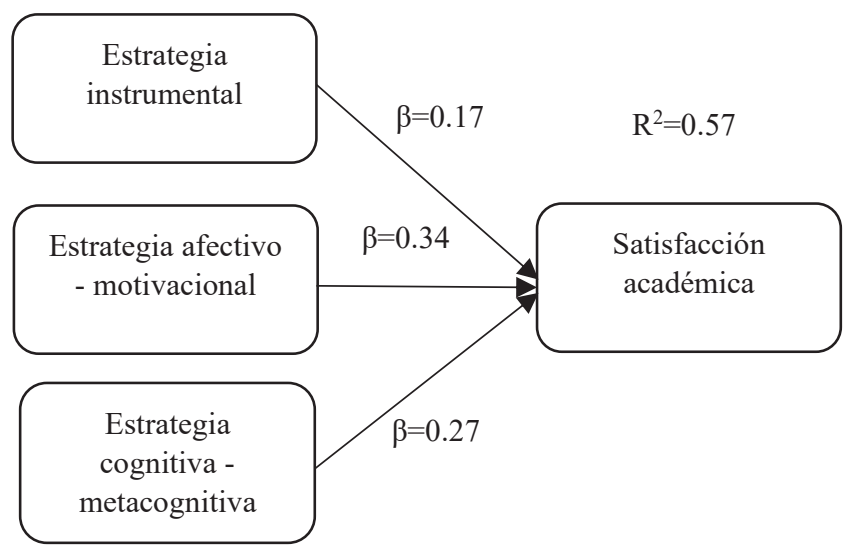

Figura 2. Resultados del modelo.

En general, el modelo global de las tres dimensiones en conjunto influye en un $56.9 \%$ en la satisfacción académica de los estudiantes universitarios en un entorno de aprendizaje virtual sincrónico.

\section{Discusión}

Los resultados obtenidos en esta investigación dan cuenta de la importancia de implementar cada una de las dimensiones en el proceso de enseñanza-aprendizaje; este aspecto trasciende si se toma en cuenta que la planificación didáctica y el ambiente del salón de clase, como indicadores de la estrategia instrumental, los cuales son claves en un proceso de enseñanza virtual sincrónica. Asimismo, los indicadores evaluativos y de mediación emocional caracterizadas en la estrategia afectivo-motivacional toman relevancia en el ámbito pedagógico, dado que, afianzan la relación docente-estudiante, convivencia en el salón de clase y formación de valores. Igualmente, la mediación cognitiva y los recursos didácticos como indicadores de la estrategia cognitivametacognitiva permiten cumplir con los objetivos académicos y la observación de la actividad cognitiva.

Tales resultados contrastan con los obtenidos por Granero et al. (2021), quienes reconocen la necesidad de establecer estrategias de enseñanza relacionados al clima motivacional en el aula, donde las relaciones interpersonales y la orientación al desarrollo de las capacidades del estudiante universitario sean de prioridad para el docente, con el fin de que los estudiantes aumenten su satisfacción y compromiso hacia la carrera. Este enfoque permite al docente universitario otorgarle autonomía y responsabilidad al estudiante sobre su propio aprendizaje, lo que promueve su implicación y disfrute en el proceso de enseñanzaaprendizaje. Cabe recalcar que, los esfuerzos educativos están direccionados a generar un alto grado de satisfacción académica, por lo que se debe estructurar tales estrategias en base a medidores que garanticen la calidad educativa y la participación activa del estudiante a lo largo de su proceso. 
No obstante, lo expuesto no es lo único que se debe considerar, pues es evidente que las condiciones de enseñanza están cambiando constantemente, considerándose como un componente dinámico, por ello, el uso de plataformas digitales y TIC's dentro de las estrategias de enseñanza no solo servirían de apoyo al desempeño docente, sino que permitirá un óptimo desarrollo en la formación universitaria online, logrando mayor interactividad entre los estudiantes y un buen entorno de aprendizaje (Cabanillas et al., 2020). Cabe recalcar que, a pesar de que la labor del docente sea implementar oportunidades de aprendizaje haciendo uso de las nuevas tecnologías, es indispensable que las entidades relacionadas a la educación universitaria empoderen a los docentes con el uso de las mismas y que puedan ser capaces de transmitir ese conocimiento a sus estudiantes, tal como lo menciona Rivas et al. (2019) en su investigación.

Como consecuencia, el docente universitario podrá ser capaz de adaptar las estrategias de enseñanza docente a un entorno de aprendizaje virtual sincrónico, ya que mantener estrategias de enseñanza tradicional, no vinculadas con los actuales estilos de aprendizaje de los estudiantes universitarios, resultaría en una baja competitividad, poca capacidad y disposición a la actualización de su saber y oficio (Ortega et al., 2019). Asimismo, permitirá al docente apostar por estrategias de enseñanza distintas a las clases magistrales, donde hará uso efectivo de los recursos didácticos en base a TIC's orientados al proceso de enseñanza-aprendizaje del estudiante.

En este mismo contexto, el ambiente universitario no considera únicamente condiciones materiales o las relaciones interpersonales básicas entre docentes y estudiantes, por el contrario, involucra vivencias de cada participante en su entorno educativo, pues va más allá de un ambiente físico, ya que mantiene mayor consideración a las acciones y experiencias que el docente genere en las clases. Como mencionan Tacca et al. (2020), esta interacción configura un sistema integrado de vínculos humanos tan complejo, no solo se limita al plano cognitivo, sino a las habilidades emocionales que el docente universitario debe mostrar para ofrecer experiencias de aprendizaje gratificantes, y que generen una alta satisfacción en los estudiantes.

Por su parte, los resultados de la satisfacción académica en el presente estudio se relacionan a los presentados por Llanes et al. (2021), quienes encontraron que la satisfacción académica vinculada a la experiencia universitaria es elevada, esto se apoya en que la motivación intrínseca de los estudiantes está relacionada a la satisfacción con la malla curricular y su relación con los docentes; además, con los resultados obtenidos por Pozuelos et al. (2021), que manifiestan que los estudiantes universitarios priorizan las estrategias aplicadas por el docente dentro y fuera de clases, considerando a las actividades relacionadas con la investigación, recursos didácticos en las plataformas virtuales, trabajos grupales y tutorías, como estrategias de enseñanza y evaluación de mayor utilidad y eficacia.

En este contexto, el buen uso de herramientas y mecanismos docentes aseguran la calidad de la enseñanza, proporcionando a los docentes un espacio de implementación de estrategias innovadoras entorno al aprendizaje del estudiante, donde se valore las interacciones humanas dentro del aula (Ruíz y Glasserman, 2021). Dicho esto, se resalta que el docente universitario puede hacer uso de su libertad de cátedra, por medio de metodologías y estrategias propias, pero, esto supone que es parte de su labor innovar en nuevas estrategias que promuevan la exploración en el aprendizaje e involucramiento para que el aprendizaje adquirido mejore las capacidades de los estudiantes.

Entre las principales limitaciones del trabajo se han identificado los siguientes; primero, se ha aplicado el cuestionario solo a la población de estudiantes de la disciplina de administración y negocios, dado su valor teórico y práctico, el cual no requiere de laboratorios para el proceso de formación; segundo, el estudio es de corte transversal, por la complejidad de realizar una investigación longitudinal en un entorno virtual.

\section{Conclusiones}

Las estrategias de enseñanza son vías utilizadas por el agente de enseñanza, comúnmente llamado docente, para promover el proceso de aprendizaje en los estudiantes. Asimismo, estas siempre desarrollan un papel intencional, pues antes de cualquier plan curricular o programa de estudio, el docente toma decisiones en torno a la manera de ejercer su conocimiento, de esta manera, guía sus acciones en base a dichas estrategias. En ese sentido, una correcta utilización no solo ayuda 
a que el proceso de enseñanza-aprendizaje se desarrolle de manera óptima, también, sirve de soporte al mantener el interés y promover el autoaprendizaje del estudiante, obteniendo mejores resultados tanto en el bienestar psicológico, disfrute de carrera y éxito académico, considerados como factores relevantes en la satisfacción académica de los estudiantes universitarios.

Como consecuencia de lo expuesto, los autores del artículo coinciden plenamente que las dimensiones de las estrategias de enseñanza docente inciden de manera positiva y significativa en la satisfacción académica. Es decir, un mayor uso de la estrategia instrumental, afectivo-motivacional y cognitivametacognitiva, inciden favorablemente en la satisfacción académica, pero, si se aplica el conjunto total de tales estrategias, los resultados son aún más favorables, permitiendo un mayor bienestar psicológico, respecto a sus experiencias en su rol de estudiante. Asimismo, se evidencia que estas estrategias deben ser desarrolladas en torno al estudiante, pues si el docente no toma en consideración las exigencias y expectativas de los mismos, este tendría serias limitaciones para poder desempeñarse con calidad, de igual manera, la constante formación del docente involucra estar preparado para cualquier reto educativo con el fin de ejercer su papel con una mayor eficiencia y eficacia.

A pesar de ello, se recomienda que estudios futuros aborden la relación entre ambas variables y se desarrollen desde un enfoque cualitativo y/o mixto en diferentes especialidades universitarias, con el fin de comparar los resultados obtenidos y lograr una comprensión holística del efecto de las variables estudiadas en el entorno universitario. Además, analizar el impacto de cada dimensión estudiada con respecto a las especialidades universitarias, con la finalidad de configurar un perfil docente en base a las exigencias de los estudiantes. También, se recomienda explorar los efectos indirectos de los factores externos e internos en el proceso de enseñanza docente y satisfacción académica del estudiante.

\section{Conflicto de intereses}

Los autores declaran no tener conflictos de interes.

\section{Referencias bibliográficas}

Abreu, Y., Barrera, A., Breijo, T. y Bonilla, I. (2018). El proceso de enseñanza-aprendizaje de los Estudios Lingüísticos: su impacto en la motivación hacia el estudio de la lengua. Mendive, 16(4), 610-623.

Beardsley, M., Albó, L.., Aragón, P., y HernándezLeo, D. (2021). Emergency education effects on teacher abilities and motivation to use digital technologies. British Journal of Educational Technology, 52(4), 1455-1477. 10.1111/bjet.13101

Betancourt, M., Bernate, J., Fonseca, I. y Rodríguez, L. (2020). Revisión documental de estrategias pedagógicas utilizadas en el área de la educación física, para fortalecer las competencias ciudadanas. Retos, 38(2), 845851. 10.47197/retos.v38i38.74918

Cabanillas, J., Veríssimo, S. y Luengo, R. (2020). Contraste en la percepción sobre el uso de una plataforma virtual para la mejora de la enseñanza y aprendizaje de las matemáticas. Revista Ibérica de Sistemas y Tecnologías de la Información, 38(09), 33-47. 10.17013/ risti.38.33-47

Candrlic, S., Jaksic, D., y Poscic, P. (2020). Do e-learning activities increase students' academic satisfaction? Proceedings of the European Conference on E-Learning, ECEL, 2020-Octob, 80-88. 10.34190/EEL.20.039

George, D., y Mallery, wP. (2019). IBM SPSS statistics 26 step by step: A simple guide and reference. 16va ed. Routledge.

Gil, M., Cordero, J. y Cristóbal, V. (2017). Las estrategias docentes y los resultados en PISA 2015. Revista de Educación, 379, 32-55.

Gonzalez, A., Pérez, C., Hechenleitner, M., Vaccarezza, G., y Toirkens, J. (2019). Satisfacción académica y prácticas pedagógicas percibidas por estudiantes de salud de Chile. Revista de La Fundación Educación Médica, 22(3), 103. 10.33588/ fem.223.992

Granero, A., Hortigüela, D., Hernando, A., y Carrasco, M. (2021). Estilo docente y competencia en Educación Superior: mediación del clima motivacional. Educación XX1, 24(2), 43-64. 10.5944/educXX1.28172 
Henríquez, P., y Arámburo, V. (2019). Análisis cualitativo en torno al uso de estrategias de enseñanza por docentes universitarios en contextos de formación de agentes educativos. Archivos Analíticos de Políticas Educativas, 27(85). 10.14507/epaa.27.3644

Hernández, R. H. y Mendoza, C. P. (2018). Metodología de la investigación: las rutas cuantitativa, cualitativa y mixta. 1ra ed. McGraw Hill México.

Hortigüela, D., Ausín, V., Delgado, V. y Abella, V. (2017). Análisis de la importancia de los criterios de evaluación y el reconocimiento académico docente universitario como indicadores de la calidad educativa en España. Revista de la Educación Superior, 46(181), 75-87. 10.1016/j.resu.2016.10.002

Hurtado, A., De la Gala, B. R., Ccorisapra, F. de M., \& Quispe, A. D. (2021). Cultura y compromiso organizacional: Efectos indirectos de la experiencia de marca empleadora. Revista Universidad y Sociedad, 13(4), 369-377. https://rus.ucf.edu.cu/index. $\mathrm{php} / \mathrm{rus} /$ article/view/2176/2155

Iglesias, I. (2016). El perfil del profesor de español como lengua extranjera: dimensión formativa, dimensión técnica y dimensión afectivoemocional. Studia Romanica Posnaniensia, 43(3) 67-83. 10.14746/strop.2016.433.005

Klimenko, O., Giraldo, P., Dereix, A. y Montaño, W. (2014). Fomento del componente cognitivo de la capacidad creativa mediante la metodología de formulación de preguntas investigativas. Revista Psicoespacios, 8(12), 378-401. 10.25057/21452776.308

Llanes, J., Méndez, J. y Montané, A. (2021). Motivación y satisfacción académica de los estudiantes de educación: una visión internacional. Educación XX1, 24(1), 45-68, 10.5944/educXX1.26491

Martínez, O., Steffens, E., Ojeda, D., y Hernández, H. (2018). Pedagogical strategies applied to education with virtual media for the generation of global knowledge. Formación Universitaria, 11(5), 11-18. 10.4067/S071850062018000500011

Orozco, A., García, T. y Cepeda, L. (2019). Alfabetización digital desde el enfoque instrumental, cognitivo y emocional en estudiantes de turismo usando blackboard.
IE Revista de Investigación Educativa de la REDIECH, 10(19), 11-35. 10.33010/ie rie rediech.v10i19.300

Ortega, E., Casanova, I., Paredes, I. y Canquiz, L. (2019). Estilos de aprendizaje: Estrategias de enseñanza en luz. Telos Revista de Estudios Interdisciplinarios en Ciencias Sociales, 21(3), 710-730. 10.36390/telos213.11

Pozuelos, F., García, F. y Conde, S. (2021). Evaluar practicas innovadoras en la enseñanza universitaria. Validación de instrumento. Educación XX1, 24(1), 69-91, 10.5944/ educXX1.26300

Rivas, B., Gértrudix, F. y De Cisneros, J. (2019). La percepción del docente universitario ante el uso y valor de los datos abiertos. Educación $X X 1, \quad 22(2)$, 141-163. Doi: 10.5944/ educXX1.21317

Ruíz, J. y Glasserman, L. (2021). Características del aseguramiento de la calidad educativa: Un mapeo sistemático 2016-2020. Revista Complutense de Educación, 32(3), 337-348. https://revistas.ucm.es/index.php/RCED/ article/view/70182/4564456558066

Tacca, H., Tacca, A. y Cuarez, R. (2020). Inteligencia emocional del docente $y$ satisfacción académica del estudiante universitario. Revista Digital de Investigación en Docencia Universitaria, 14(1), 1-16. 10.19083/ridu.2020.887

Vergara-Morales, J., Del Valle, M., Díaz, A. y Peréz, M. (2018). Adaptación de la Escala de Satisfacción Académica en Estudiantes Universitarios Chilenos. Psicología Educativa, 24(2), 99-106. 10.5093/ psed2018a15

Zambrano L. (2020). Uso de la Tecnología de la Información y Comunicación en educación virtual y su correlación con la Inteligencia Emocional de docentes en el Ecuador en contexto COVID-19. Revista Ibérica de Sistemas y Tecnologías de la Información, 40(12), 31-44. DOI: 10.17013/risti.40.31-44

Zambrano, R., Gil, N., Lopera, E., Carrasco, N., Gutiérrez, A. y Villa, A. (2015). Validez y confiabilidad de un instrumento de evaluación de estrategias docentes en la práctica de la enseñanza universitaria. Magister, 27(1), 2636. 10.1016/j.magis.2015.06.002 
Anexo 1. Instrumento de recolección de datos

\section{Estrategia instrumental}

Ítem 1: Fomenta la participación de los estudiantes en la clase presencial / virtual

Ítem 2: Tiene en cuenta las diferencias individuales de los estudiantes

Ítem 3: Orienta el aprendizaje de manera respetuosa y amable

Ítem 4: Hace cumplir normas de convivencia para el desarrollo de la clase

Ítem 5: Demuestra interés por el aprendizaje de sus estudiantes

Ítem 6: Informa oportunamente sobre las diversas actividades del curso

Ítem 7: Presenta el cronograma de las actividades y formas de evaluación

Ítem 8: Explica lo que se espera que el estudiante logre con el curso

Ítem 9: Formula con claridad los criterios de rendimiento esperados para cada una de las actividades

\section{Estrategia afectivo-motivacional}

Ítem 1: Valora los aportes de los estudiantes al desarrollo del curso

Ítem 2: La evaluación estimula la comprensión y aplicación de lo aprendido

Ítem 3: Incentiva la argumentación en las evaluaciones

Ítem 4: Utiliza diferentes formas de evaluación

Ítem 5: Es equitativo en los criterios de evaluación

Ítem 6: Retorna los trabajos y evaluaciones con las observaciones pertinentes

Ítem 7: Toma decisiones a partir de la evaluación para reorientar el aprendizaje de los estudiantes

Ítem 8: Los aprendizajes evaluados se relacionan con las competencias del curso

Ítem 9: Retorna oportunamente los trabajos evaluados

Ítem 10: Respeta la dignidad de las personas

Ítem 11: Respeta los argumentos de los estudiantes

Ítem 12: El docente controla sus emociones

Ítem 13: Sabe mantener la disciplina en las sesiones de clase

Ítem 14: Estimula positivamente la expresión de argumentos de los estudiantes

Ítem 15: Logra un ambiente agradable en el desarrollo de la clase 
Ítem 16: Estimula a los estudiantes al trabajo interdisciplinario

\section{Estrategia cognitiva-metacognitiva}

Ítem 1: Elabora guías de aprendizaje para los estudiantes

Ítem 2: Presenta asuntos de actualidad en el desarrollo de los temas

Ítem 3: Promueve el aprendizaje mediante la solución de problemas

Ítem 4: Adapta la enseñanza a los ritmos de aprendizaje de los estudiantes

Ítem 5: Respalda la enseñanza con procesos y resultados de investigación

Ítem 6: Usa otras formas de explicación cuando los estudiantes no entienden un tema

Ítem 7: Promueve la integración con otras áreas

Ítem 8: Se apoya en el uso de tecnologías de información (TIC) para el desarrollo del curso

Ítem 9: Recurre a diferentes fuentes de información para el desarrollo del curso.

Ítem 10: Estimula la consulta de diversas fuentes de información

\section{Satisfacción académica}

Ítem 1: Estoy satisfecho con la decisión de haber elegido este programa de estudios

Ítem 2: Me siento cómodo con el ambiente de aprendizaje generado en los cursos del programa de estudios

Ítem 3: Disfruto de mis clases la mayor parte del tiempo.

Ítem 4: En general estoy satisfecho con mi experiencia académica.

228 Ítem 5: Disfruto cuando me estimulan intelectualmente en las asignaturas del programa de estudios Ítem 6: Me entusiasman los contenidos transmitidos en las asignaturas del programa de estudios Ítem 7: Me gusta lo que he aprendido en las asignaturas del programa de estudios 